\title{
Unusual bone metastasis from follicular carcinoma thyroid: presenting as a primary bone malignancy
}

\author{
Saurabh Varshney ${ }^{1}$, Harish Chandra Pant ${ }^{2}$ \\ ${ }^{I}$ Department of E.N.T. \& Head Neck Surgery, All India Institute of Medical Sciences, Rishikesh, Uttarakhand, India \\ ${ }^{2}$ Department of Radiology, Himalayan Institute of Medical Sciences, Dehradun, India
}

Received August 26, 2014; Revised September 30, 2014; Accepted October 01, 2014; Published Online October 02,2014

\section{Case Report}

\begin{abstract}
Osseous metastasis in patients of thyroid carcinoma is not very common, and appendicular skeleton is very rare. Bone metastasis represents a complication, especially of follicular thyroid carcinoma and severely reduces the quality of life causing pain, and fractures. Diagnosis is typically done by correlating clinical suspicion with imaging. Occult clinical presentations usually delay the detection and management of patients with bone metastasis from thyroid carcinoma. There is limited information about the clinical presentations and prognosis of patients with follicular thyroid carcinoma with bone metastasis. We hereby present a case report of right arm swelling diagnosed as metastatic carcinoma having metastasis from thyroid primary.
\end{abstract}

Keywords: Follicular Carcinoma; Thyroid; Skeleton Metastasis

\section{Introduction}

Metastatic tumors of bone are uncommon and a thyroid origin is considered exceedingly rare. Differentiated thyroid cancer presenting with osseous metastases is very rare as it is reported in 3-12\% cases only. ${ }^{1,2}$ Out of the two differentiated thyroid cancers, higher prevalence of bone metastasis occurs in follicular $(15.2 \%)$ than in papillary carcinoma $(0.6 \%)$. $^{2,3}$ Although rare, the common sites of osseous metastases are sternum, vertebrae, pelvis, and ribs, while metastases to appendicular skeleton is the rarest., ${ }^{1,2}$ A case of metastatic follicular thyroid carcinoma presenting as a painful swelling in the right arm of a 60-year-old female is reported here.

\section{Case Report}

A 60-year-old woman presented with progressively increasing swelling over right upper arm [Figure 1] with mild pain and restriction of movements for 8 months. She also suffered from dyspnoea on exertion. During examination, her general condition was low. She had a large swelling over right upper arm extending from the shoulder to just above the elbow, firm to hard in consistency, non-tender, with dilated cutaneous veins and superficial ulceration. She did not have regional lymphadenopathy. Laboratory Investigations revealed raised ESR. (Figure 1)

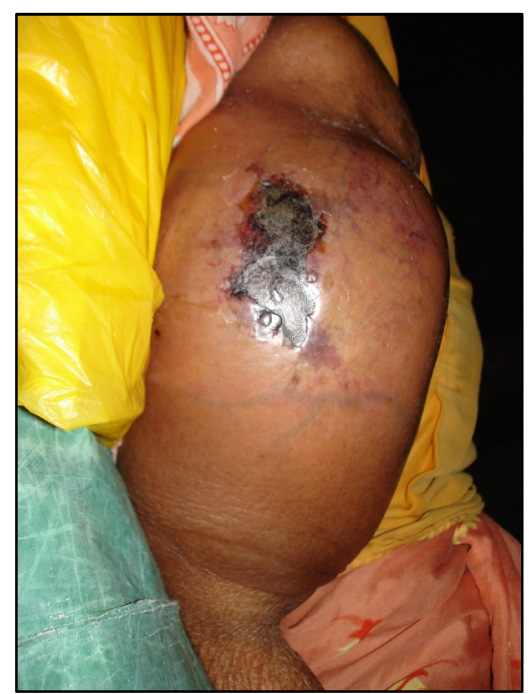

FIG. 1: Soft tissue swelling with ulceration of right upper arm.

\section{Radiological Examination}

Radiograph Right Arm AP/Lateral [Figure 2] showed an expansile and destructive lesion in the diaphysis and metaphysis of humerus with few thinned remaining bony trabeculae, mostly in the proximal part, and an associated large lobulated soft tissue swelling without any evidence of calcification. However, in spite of the massive extent of destruction of bone, the proximal and distal epiphyseal regions appeared to 
be reasonably preserved. Radiograph Chest PA View [Figure 3] showed multiple rounded opacities with well-defined margins in both lung fields, suggestive of secondaries.

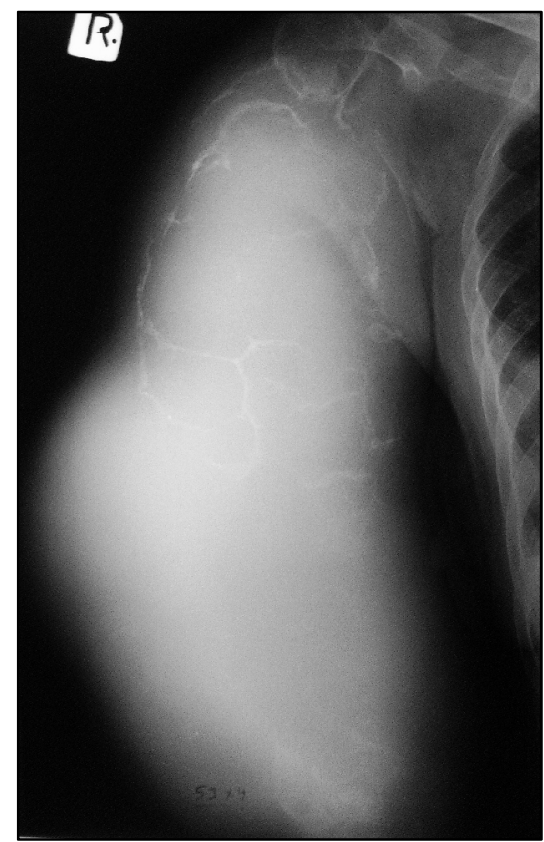

FIG. 2: Radiograph right arm AP shows an expansile destructive lesion in the diaphysis with a large soft tissue swelling.

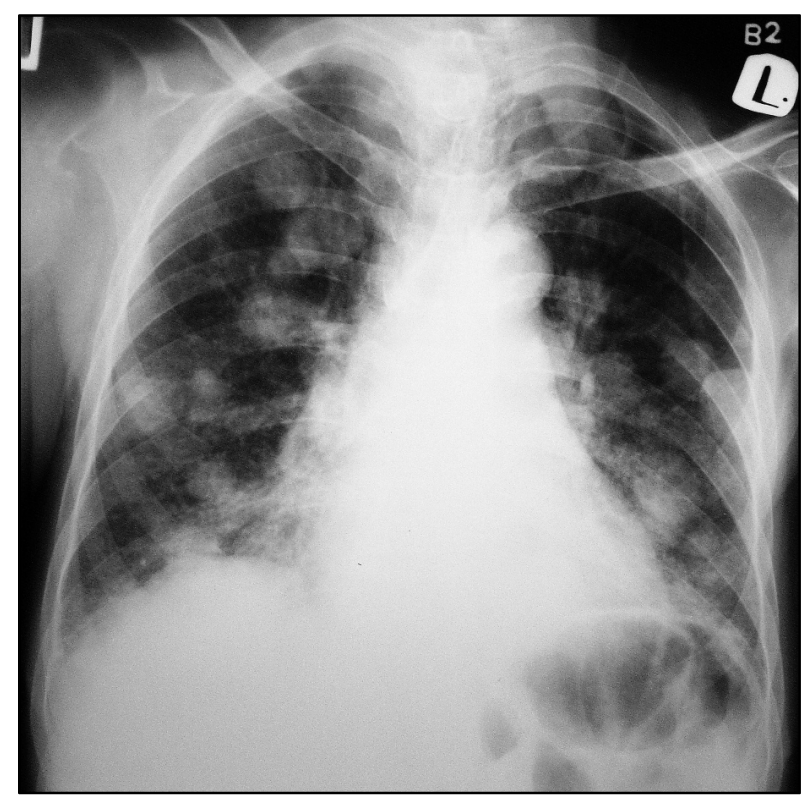

FIG. 3: Radiograph chest PA view reveals multiple rounded well-defined opacities in both lungs.

Non-contrast CT right arm [Figure 4] revealed massive destruction of the normal osseous anatomy by a large heterogenous isodense mass lesion, involving the metadiaphysis of humerus and displacing the few remaining thinned bony trabeculae peripherally, with a contiguous large extra osseous component, devoid of any calcification, hemorrhage or necrosis. Based on these radiographic findings of a large de- structive bone tumor with a prominent soft tissue component and pulmonary metastasis, a provisional radiological diagnosis of primary malignant bone tumor was kept, probably fibro/angiosarcoma or malignant fibrous histiocytoma.

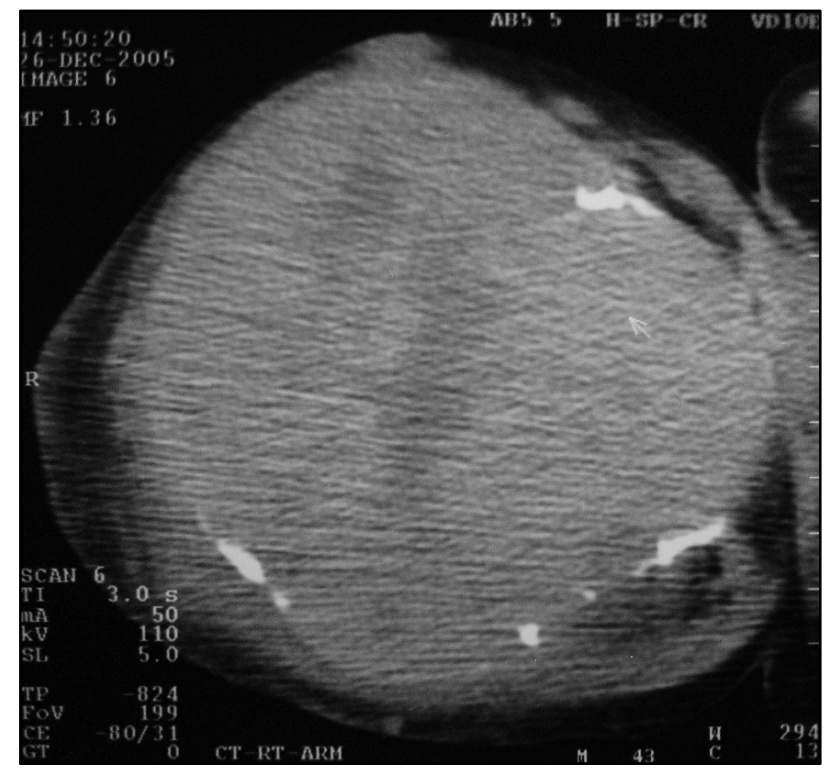

FIG. 4: CT right upper arm reveals massive destruction of the bone with thinned bony trabeculae peripherally.

\section{Histopathological Examination}

Fine needle aspiration cytology (FNAC) from the humeral mass showed small and large loosely cohesive clusters of cells with mild anisonucleosis and scanty to moderate amount of pale blue-grey cytoplasm with pink periphery [Figure 5]. At many places, these clusters were seen to be arranged in acinar and tubular patterns. Mitotic figures were not seen. A background of necrotic debris, abundant cystic macrophages and degenerated RBCs were present along with regions having amorphous bluish colloid material. These findings suggested an epithelial meatastatic carcinoma, probably from a primary in thyroid.

After this perplexing finding, the patient was re-examined and small inconspicuous nodules were found in the thyroid on clinical examination. High resolution Ultrasound (HRSG) Neck [Figure 6] of the patient was then performed which revealed small heterogenous hyperechoic nodules with calcification and slight increased vascularity. Subsequently, USG guided FNAC from thyroid showed features suggestive of malignancy of thyroid, probably follicular carcinoma. 

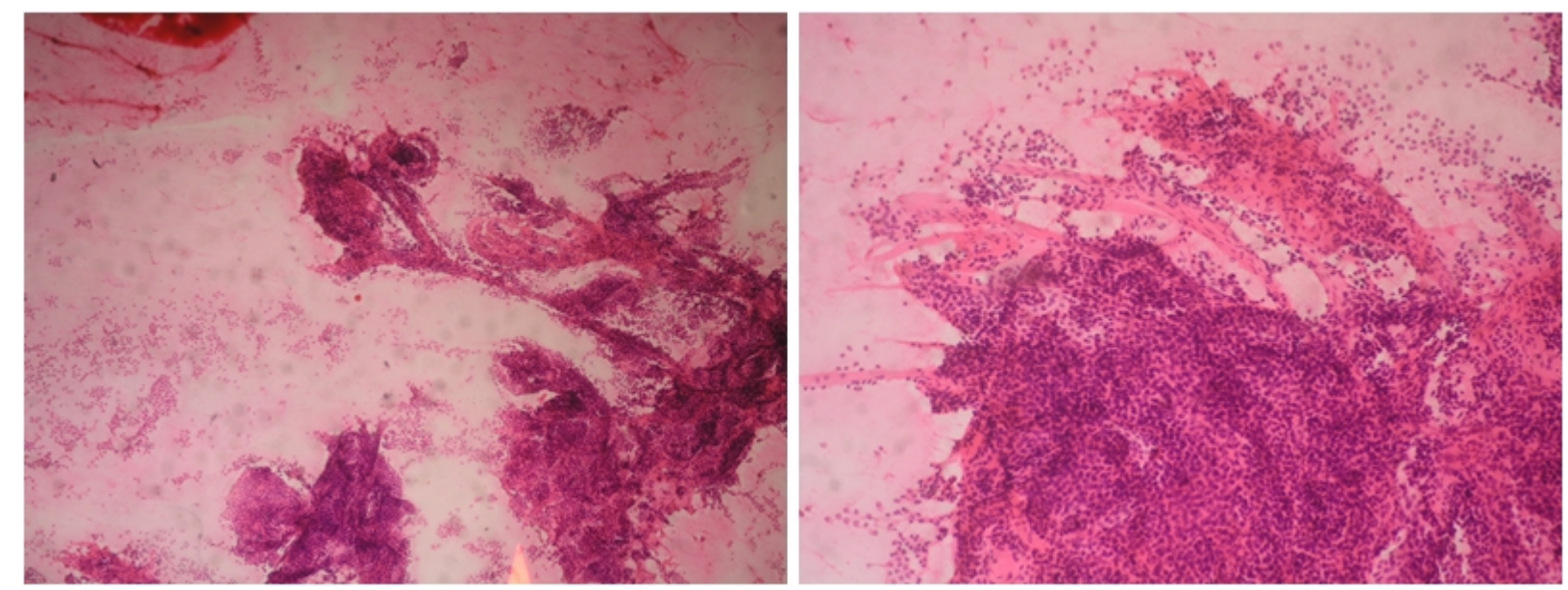

FIG. 5: Fine needle aspiration cytology of right arm swelling.
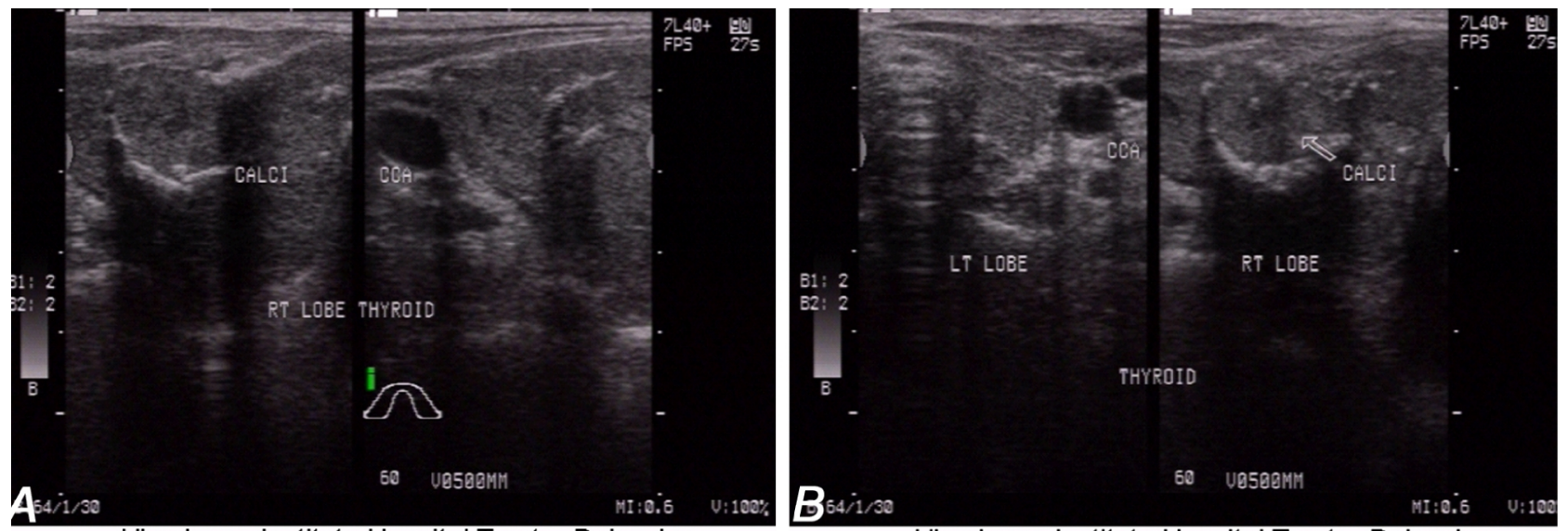

Himalayan Institute Hospital Trust, Dehradun.

Himalayan Institute Hospital Trust, Dehradun.

FIG. 6: Ultrasound Neck shows small heterogenous hyperechoic nodules.

\section{Discussion}

The present case has two interesting features: (1) a relatively uncommon site of bone metastasis and (2) an uncommon physical and radiological appearances of the osseous secondary. In one of the largest available series of 45 cases of differentiated thyroid carcinoma who had bone metastasis at presentation, sternum was the most frequent site. ${ }^{1}$

Metastasis in the bone is the third leading site for metastasis after lung and liver; about $15 \%$ of all carcinoma clinically manifest bone metastasis. ${ }^{3}$ The majority of the bone metastasis are caused by cancer of the breast, lung, followed by thyroid as an intermediate group. ${ }^{3}$ In the limbs, the incidence decreases from proximally to distally but in our case, the whole shaft of the right humerus was involved, which is unusual for bone metastasis and mimicked primary bone tumor. The main symptoms like pain and pathological fracture were not seen but large smooth soft tissue swelling of whole of the right upper arm was noted, which appeared to be rather unusual for metastasis, and more suggestive of primary bone tumor.

The prominent soft tissue mass is not an early feature of metastatic bone disease as it is in most sarcomas of bone, but it can be seen in advanced stages of metastasis. New modalities like spiral CT, MRI are used to reveal the primary site. CT can be used to know the extent of mass and calcification, and to guide FNAC. USG screening of the primary site, especially thyroid and kidney, is very important as it reveals the primary site of tumor along with vascularity. In this case, the FNAC was done from the right upper arm soft tissue swelling which showed metastatic lesion. Primary search was done especially for thyroid. HRSG of thyroid showed a heterogenous echogenic mass with increased vascularity of the right lobe. Doppler study showed increased vascularity, suggestive of malignancy of thyroid. Repeat biopsy from thyroid was done which showed a primary thyroid carcinoma, possibly follicular in nature. The histology of the metastatic deposits usually repeats the histology of primary carcinoma. In most 
cases, however, the organ of origin of the metastasis cannot be determined by histological examination. ${ }^{4}$

Bone metastasis is purely lytic, blastic, and mixed. Bone metastasis is purely lytic as found in liver, breast and thyroid. ${ }^{5}$ Secondaries from carcinoma of the thyroid are usually associated with pure osteolysis and blow out appearances of the bone. ${ }^{6}$ Lytic metastasis may remain occult in conventional radiographs until it has completely destroyed bone trabeculae and reached 2-3 cms diameter in size. ${ }^{7}$ The characteristic of lytic lesion is that of indistinct border with permeation of the cortex or erosion and breakdown. The periosteal reaction is absent.

The most frequent and typical metastasis presents with follicles lined by small cubical cells and containing colloid (follicular carcinoma). Papillary, medullary and anaplastic carcinoma are less frequently encountered in bone metastasis from the thyroid. ${ }^{7}$

The rate and location of metastatic spread varies according to the histologic subtype of thyroid cancer. Up to $6 \%$ of patients with well-differentiated thyroid carcinoma have been reported to develop distant metastasis, with a predilection for bones (43\%) and lung (40\%). ${ }^{8}$

In this case, the bone metastasis was the presenting symptom, the primary being silent. Radiological imaging of purely osteolytic metastasis may mimic fibro/angio sarcoma or multiple myeloma. Sometimes, a metastasis from thyroid may mimic a giant cell tumor due to abundant production of reactive osteoclast-like cells. ${ }^{6}$ This is an exceptional case of follicular carcinoma of thyroid metastasizing to the humerus in our study.

A diagnostic workup of bone metastasis includes proper clinical evaluation, different radiological modalities which include conventional radiograph of the skeletal site, CT and MRI of the osseous lesions. Ultrasound for thyroid and kidney including prostate should be done to rule out the primary site, as it is the quick non- invasing and non-ionizing modality. All these investigations should be followed by biopsy of the bone lesion.

\section{Conflict of interest}

The authors declare that they have no conflicts of interest. The authors alone are responsible for the content and writing of the paper.

\section{References}

1. McCormack KR. Bone metastases from thyroid carcinoma. Cancer 1966; 19:181-4.

2. Marcocci C, Pacini F, Elisei R, et al. Clinical and biologic behaviour of bone metastases from differentiated thyroid carcinoma. Surgery 1989; 106:960-6.

3. Galasko CSB. Detection of Skeletal Metastasis. J Bone Joint Surgery 1971; 53-B: 153.

4. Greenspan A, Norman A. Osteolytic cortical destruction: an unusual pattern of skeletal metastases. Skeletal Radiol 1988; 17:402-6.

5. Jacobsen S, Stephensen SL, Paaske BP, et al. Skeletal metastases of unknown origin: a retrospective analysis of 29 cases. Acta Orthop Belg 1997; 63:15-22.

6. Soderlund V. Radiological Diagnosis of Skeletal Metastases. Eur Radiol 1996; 6: 587-95.

7. Muresan MM, Olivier P, Leclère J, et al. Bone metastases from differentiated thyroid carcinoma. Endocr Relat Cancer 2008; 15:37-49.

8. Wood WJ Jr, Singletary SE, Hickey RC. Current results of treatment for distant metastatic well-differentiated thyroid carcinoma. Arch Surg 1989; 124:1374-7. 